\title{
Übersetzungsäquivalente substantivierter Adjektivwörter vom Typ das Neue, das Schöne (neutrale Abstrakta) im Polnischen
}

\begin{abstract}
The article discusses the translation problem of adjectives converted into nouns such as das Neue, das Moderne. There are presented results of research based on the corpus of more than 400 entities and their translations from German into Polish. The analysis used tools of Cognitive Linguistics to catch most important semantic differences between equivalents. Five types of equivalents are the most frequently chosen but only 20 per cent solutions in Polish are adjectives converted into nouns. Therefore the supposition is that these formations can be the source of difficulties for translators.
\end{abstract}

\section{VORBEMERKUNGEN}

Dem vorliegenden Artikel liegt eine Untersuchung zugrunde, in der $\ddot{\mathrm{u}}$ ber 400 substantivierte Adjektivwörter ${ }^{1}$ vom Typ das Neue, das Abgeschmackte und ihre Übersetzungen analysiert wurden. ${ }^{2}$ Das Material wurde in seinem überwiegenden Teil Adrianne von Speyrs Beichte entnommen und durch Exzerpte aus anderen Quellen ergänzt.

Da unter dem Terminus Substantivierung in der einschlägigen Literatur Verschiedenes gemeint ist, soll er anfangs definiert werden. Unter Substantivierung wird in diesem Beitrag sowohl der Wortbildungsprozess als auch sein Produkt verstanden. Im Falle einer Substantivierung wird ein Konzept so ausgedrückt, dass auf eine begriffliche Inhaltseinheit, die konventionell kein Substantiv ist, ein Substantivschema aufgelegt wird, ohne dass mor-

1 Zum Terminus substantivierte Adjektivwörter vgl. Jodłowski (1964).

2 Vgl. Kubaszczyk, Substantywizacje w przekładzie. Niemiecko-polskie studium kontrastywne. Poznan 2003 (unveröffentlichte Dissertation). 
phologische Änderungen der Basis eintreten. In dem Sinne sind solche Wortbildungen wie das Neue oder das Schreiben Substantivierungen, dagegen die Formationen Behandlung oder Neuheit zwar Nominalisierungen, aber keine Substantivierungen.

Die Klasse der substantivierten Adjektivwörter ist nicht homogen. Im Folgenden werden unter der Bezeichnung substantiviertes Adjektivwort mehrere Subklassen subsumiert und zwar: (1) in prädikativer Form substantivierte Adjektive, unflektiert, (2) in attributiver Form substantivierte Adjektive, die ihre adjektivische Flexion beibehalten, hierzu rechnen auch die als Positiva, Komparativa und Superlativa substantivierten Adjektive, (3) in prädikativer Form substantivierte Adjektive, die die substantivische Flexion angenommen haben, (4) substantivierte Partizipien, (5) substantivierte Gerundiva, (6) substantivierte Ordinalzahlen.

Substantiviert werden sowohl einzelne Adjektivwörter als auch aus mehreren Adjektivwörtern bzw. einem Adjektivwort und einer anderen Wortart bestehende Wortgruppen. Substantivierte Adjektivwörter können selbständig oder mit verschiedenen Determinativen vorkommen. Die Determinative können die Übersetzung beeinflussen.

Die theoretische Grundlage der Untersuchung bildet das Theoriegut der kognitiven Linguistik, die in R. W. Langacker ihren Hauptvertreter hat. Seine Theorie wurde in zahlreichen Aufsätzen und Monographien dargestellt, für die vorliegende Betrachtung ist vor allem die Arbeit Concept, Image, and Symbol (1991) von Bedeutung. Der Sprachnutzer wird in der kognitiven Theorie als Konzeptualisator oder Konzeptualisierender bezeichnet. Die kognitive Linguistik geht davon aus, dass die Sprachteilhaber die Wirklichkeit auf viele verschiedene Weisen wahrnehmen können, was in Konsequenz zu vielen in unterschiedlichen Sprachbildern erfassten Konzeptualisierungen führt. In der Übersetzung kann es zu Änderung eines kreierten Textbildes kommen. In diesem Aufsatz wird auf solche Termini wie Trajektor (TR), Landmark (LM), Vordergrundfigur und Profilierung zurückgegriffen. Die Profilierung erfolgt nach Langacker auf allen sprachlichen Ebenen. Das Profil einer prädikativen Struktur ist „a substructure elevated to a special level of prominence within the base, namely that substructure which the expression 'designates'" (Langacker 1991: 5). Während die einen Elemente durch die Profilierung hervorgehoben werden und den Status der Figur erlangen, fällt den anderen Strukturen die Rolle des Hintergrunds zu (eng. figure and ground organization). Trajektor und Landmark sind Elemente, die eine Relation bilden. Trajektor ist Vordergrundfigur im Relationsprofil einer semantischen Struktur und Landmark eine andere Substruktur, eine Hintergrundfigur, die neben Trajektor im Relationsprofil hervorgehoben wird (vgl. Taylor 2002: 592). 


\section{DIE FREQUENZ DER SUBSTANTIVIERTEN NEUTRALEN ADJEKTIVWÖRTER IM DEUTSCHEN UND IM POLNISCHEN}

Im Deutschen können adjektivische Bezeichnungen aller Gegenstände und Begriffe substantiviert werden. Dieselbe potentielle Möglichkeit stellt für das Polnische Stanisław Jodłowski (1964: 44) fest. Die Substantivierung von adjektivischen Bezeichnungen der Gegenstände und Begriffe ist im Polnischen jedoch nicht so weit verbreitet wie im Deutschen, was die folgenden Stichproben eindeutig erkennen lassen. In der ersten Stichprobe wurden deutsche Ausgangstexte (AT) (aus den Bereichen Literatur, Philosophie und Theologie) mit ihren Übersetzungen verglichen:

\begin{tabular}{|l|c|c|}
\hline \multicolumn{1}{|c|}{ Untersuchter Text } & \multicolumn{1}{|c|}{$\begin{array}{c}\text { Deutscher Text } \\
\text { Anzahl }^{3}\end{array}$} & $\begin{array}{c}\text { Polnischer Text } \\
\text { Anzahl }^{4}\end{array}$ \\
\hline Doktor Faustus, Kapitel XIII & $69(1)$ & 0 \\
\hline $\begin{array}{l}\text { Sittliche Grundhaltungen / } \\
\text { Fundamentalne podstawy moralne, } \\
\text { 4. Kapitel }\end{array}$ & $44(1)$ & 4 \\
\hline $\begin{array}{l}\text { Nacholge / Nasladowanie, } \\
\text { Kapitel Der Feind - Das "Außeror- } \\
\text { dentliche" / Nieprzyjaciel - "nad- } \\
\text { zwyczajnosc" }\end{array}$ & $45(0)$ & 0 \\
\hline
\end{tabular}

Auch in den ursprünglich polnischen Texten ist die Anzahl der festgestellten Substantivierungen auffallend geringer:

\begin{tabular}{|l|c|}
\hline \multicolumn{1}{|c|}{ Untersuchter Text } & Anzahł \\
\hline $\begin{array}{l}\text { J. Tischner, Spór o istnienie człowieka, Kap. Czas ciała - } \\
\text { czas czlowieka, S.101-124 }\end{array}$ & 12 \\
\hline $\begin{array}{l}\text { M. Grabowski, Na obrzezach sacrum, Kap. Uwiedzenie, } \\
\text { S. } 12-15\end{array}$ & 3 \\
\hline $\begin{array}{l}\text { T. Węclawski, W teologii chodzi o Ciebie, Kap. List 1: } \\
\text { O wierze w wiarę, S. 37-43 }\end{array}$ & 3 \\
\hline
\end{tabular}

Dafür kommen bei allen Autoren und Übersetzern Formationen vor, die nach den Ergebnissen der Untersuchung polnische Äquivalente der deutschen neutralen Adjektivsubstantivierungen sind, wie etwa "tragizm, jakies dobro, sprawa poważna, autentyczność, wymiar zabawy" (Tischner), "zbiór spraw wazkich $i$ cennych, czegoś, co wiele warte" (Grabowski), "to, co widać, to, co jest gtębiej, niezwykłe sprawy, coś odwrotnego" (Węclawski). Diese Äquivalente werden jetzt im Einzelnen besprochen.

${ }^{3}$ Darunter in Klammern Bildungen vom Typ etwas Neues, nichts Rechtes.

${ }^{4}$ Bildungen vom Typ cos / nic nowego werden im Polnischen nicht als Substantivierungen betrachtet, deswegen werden sie durch die Statistik nicht erfasst. 


\section{3. ÄNDERUNG DER WORTBILDUNGSSTRUKTUR IM RAHMEN DERSELBEN WORTART}

Die Änderung der Wortbildungsstruktur im Rahmen derselben Wortart war mit 36,7\% aller Entscheidungen die am häufigsten gewählte Übersetzungslösung. $\mathrm{Zu}$ dem Typ wurden alle Fälle gerechnet, wo der Übersetzer bei dem Substantiv bleibt, es aber nicht mit einer Substantivierung im Zieltext (ZT) wiedergibt. Hierzu gehören u.a. mit solchen Suffixen abgeleitete Nomen wie -ość, -stwo, -ota, -izna, etc., vgl.:

(1) (...) das Barbarische sieht (...). (DF, 292),

(...) dopatruje się barbarzyństwa (...). (DF, 201);

(2) (...) Vereinigung des Schusterbubenhaften, das nun einmal dieser Technik anhaftet, mit dem künstlerisch Ernstzunehmenden (...). (DF, 349),

(...) połączenie nieodłącznego od tej właśnie techniki sowizdrzalstwa $z$ artystyczną powagą (...). (DF, 240);

(3) (...) ohne Beziehung zum Bösen und Häßlichen (...). (DF, 141),

(...) w żadnym stosunku do zła i brzydoty (...). (DF, 99);

(4) das Wirkliche (DF, 227) - rzeczywistość (DF, 158).

Am stärksten war das Suffix -ość vertreten $(9,4 \%$ aller Übersetzungslösungen), was der allgemeinen Tendenz im Polnischen entspricht. Jadacka (2001: 142) stellt fest, dass die Ableitungen mit der Nachsilbe -ość im Polnischen völlig kategorial sind 5 . Es besteht die Möglichkeit, mit diesem Suffix von fast allen Adjektiven ein Substantiv abzuleiten, bei neueren Formationen kommt fast ausschließlich dieses Suffix zum Einsatz.

\section{ETIKETT}

Im Falle dieser Übersetzungslösung wird die Bedeutung der entsprechenden Substantivierung auf zwei sprachliche Zeichen verteilt, wir haben hier also mit der Dilution ${ }^{6}$ zu tun. Die Substantivierung wird mit Hilfe einer aus einem Adjektiv (meistens) und einem Substantiv bestehenden Nominalphrase wiedergegeben, wobei das Adjektiv (oder ein substantivisches Attribut zu dem Etikett) die eigentliche Bedeutung trägt. Das Etikett ist ein Substantiv, das meist eine ganz allgemeine Bedeutung hat. Es kann aber auch Etiketts mit einer konkreten Bedeutung geben, sie werden dann als konkre-

${ }^{5}$ Grzegorczykowa (1979: 34) sprach vor 20 Jahren noch davon, dass diese Bildungen fast kategorial seien.

6 Vgl. Vinay/Darbelnet (1958/60) und Malblanc (1961, 1968). 
tisierende Etiketts bezeichnet. Übersetzungstechnisch wird hier die Substantivierung auf ein ihr zugrunde liegendes Adjektiv zurückgeführt und ein Substantiv hinzugefügt. Die Wahl des Substantivs erfolgt aus einer bestimmten Menge, ist aber nicht beliebig7. Vielmehr hängt sie stark von den Textinstruktionen ab. Diese sind $u$. a. das globale Konzept, das in einem Text/Textabschnitt realisiert wird, die Funktion der Substantivierung (z. B. die binär-kategorisierende Funktion als eine der Voraussetzungen für die Selektion des Etiketts rzeczy), die Semantik des regierenden Verbs, die Semantik der Wortbildungsbasis etc.

Die wichtigsten allgemeinen - substantivierenden - Etiketts im Polnischen sind: element, rzeczywistość, sprawa, charakter, wymiar, płaszczyzna, przypadek, sytuacja, strona, rzecz, moment, sfera, proces, stan, forma, fakt, pierwiastek. Hier zwei Beispiele für den Einsatz dieses Äquivalenztyps:

(5) (...) der (...) aus dem bloß Musikalischen ins allgemein Geistige hinaustretenden Kunst (...). (DF, 192);

(...) która ze spraw czysto muzycznych wkracza w ogólne, intelektualne sfery (...). (DF, 134);

(6) (...) viel Schmeichelhaftes (...). (DF, 160),

(...) wiele elementów pochlebnych (...). (DF, 112).

Bei den konkretisierenden Etiketten interpretiert der Übersetzer die zu übersetzende Einheit und engt ihre Bedeutung auf einen bestimmten Bereich ein, wodurch Vagheit und Unbestimmtheit der Ausgangsstruktur in der Regel aufgehoben werden (das Fruchtbare / żyzna gleba). Die unten stehenden Beispiele veranschaulichen, wie die Konkretisierungen von der Übersetzerin, Małgorzata Łukasiewicz, aus dem engen Kontext abgeleitet wurden:

(7) (...) rochen in diesem Brei nichts Besonderes mehr (...). (P, 44),

(...) nie czuli szczególnego zapachu tej mgly (...). (P, 36);

(8) (...) so daß Grenouille zögerte, seinen Geruch aufzuspalten in das Fischige, das Salzige, das Wäßrige, das Tangige, das Frische und so weiter $(P, 46)$,

(...) totez Grenouille wahał się przed rozłozeniem go na zapach ryb, soli, wody, trawy morskiej, świeżości i tak dalej $(P, 37)$.

Das letzte Beispiel zeigt auch, dass Adjektive zwar die meist gebrauchten Attribute zu dem Etikett sind, aber nicht die einzig möglichen.

Dieser Übersetzungstyp umfasst 23,9\% aller Lösungen und ist somit rein quantitativ als der zweitwichtigste $\mathrm{zu}$ gewichten.

${ }^{7}$ Diesen Äquivalenztyp bespreche ich ausführlich im Artikel Übersetzung substantivierter Adjektive als Sachnamen ins Polnische mit Hilfe eines Etiketts, Convivium 2004. S. 245-262. 


\section{NOMINALISIERUNGSKONSTRUKTION MIT "TO, CO"}

$\mathrm{Zu}$ diesem Typus wurden Fälle gerechnet, in denen die Substantivierung mit einer durch das "to, co" eingeführten periphrastischen Struktur ins Polnische übersetzt wurde. Diese Struktur ist zugleich die Wortbildungsparaphrase der jeweiligen Substantivierung, vgl. das Neue - das, was neu ist - to, co nowe. Im strengen Sinne geht es hier um syntaktische Nominalisierung: in die Struktur des Hauptsatzes wird mit Hilfe des Pronomens co (Einleitewort) ein attributiver Nebensatz oder - bei Ellipse des Verbs - eine subordinierte attributive Äußerung eingeführt. Der Exponent der grammatischen nominalen Kategorie ist in der syntaktischen Nominalisierung das Demonstrativpronomen to. Dieses Schema ermöglicht es, den Satz in der für ein Substantiv vorgesehenen Position zu gebrauchen. Die Konstruktion to, co... wurde im untersuchten Korpus insgesamt in 8,8\% der Fälle verwendet.

Die syntaktischen Nominalisierungen, welche durch den Nominalisierungsrahmen to, co eingeleitet werden, können morphologisch dreierlei Struktur aufweisen: die subordinierte Äußerung kann ein einfaches (to, co rozumiem) oder ein komplexes aus dem Kopulaverb być (sein) und einem adjektivischen Prädikativ bestehendes Prädikat (to, co jest gtębiej) enthalten. Am stärksten verbreitet sind jedoch elliptische Nominalisierungen, in denen das Kopulaverb być ausgelassen wurde (to, co piękne). Sie entsprechen auch am genauesten den deutschen Adjektivsubstantivierungen. Sie lassen denselben Grad an semantischer Unbestimmtheit und Vagheit profilieren, den die deutschen Substantivierungen aufweisen, vgl.:

(9) (...) Ungeduld, Unzufriedenheit (...). Kleinliches, Erbärmliches, das sie gebeichtet haben, weil man eben beichten muß. (B, 123),

(...) niecierpliwość, niezadowolenie (...). To, co źałosne i małostkowe, bo wlaśnie $\mathrm{z}$ tego trzeba się spowiadać. $(\mathrm{S}, 93)$.

Die Konstruktion "to, co... + jest .... Adjektiv" kommt in erster Linie da vor, wo der Übersetzer die übersetzte Substantivierung zum vollen Satz ausbaut, indem er meistens auch zusätzliche Informationen hinzufügt (unterstrichen mit Wellenlinie):

(10) Jeder weiß, daß in der Beichte des andern das UnteilbarGemeinsame mit zur Sprache kommen kann (...). (B, 157),

Każde $\mathrm{z}$ nich wie, że w spowiedzi moźe być omawiane to, co $\mathrm{w}$ małżeństwie jest niepodzielnie wspólne (...). (S, 119);

(11) Die Unterscheidung zwischen dem Subjektiven und dem Objektiven ist in der doppelten Sündenerkenntnis des Herrn grundgelegt. (B), Podstawę rozróżnienia między tym, co tutaj jest subiektywne i obiektywne, stanowią dwa rodzaje Jezusowego poznania grzechów. (S) 
Übersetzungstechnisch ist die dritte Variante am interessantesten, denn hier liegt das Moment der Interpretation. Bei der Konstruktion "to, co... + V" fällt zunächst auf, dass relativ viele substantivierte Partizipien II mit ihrer Hilfe übersetzt wurden ( $25 \%$ aller substantivierten Partizipien II). Bei der Überführung in das Verb wurden im Polnischen überwiegend Vergangenheitstempus und der vollzogene Aspekt gewählt. Die Wahl der Person wurde in den meisten Fällen vom Kontext gesteuert, im untersuchten Material war es meistens die 3. Person Singular:

(12) (...) das Eingesehene würde mir helfen (...). (B, 15), To, co zrozumiem, dopomoże mi zacząć życie od nowa. $(S, 14)$;

(13) Anderseits darf er dem Gehörten nicht auf gefährliche Art nachhängen. (B, 146);

(14) (...) zadręczania się tym, co słyszał (...). (S, 110)

In allen Übersetzungsfällen kam es zur Konversion des Passivs zum Aktiv, vgl.: das (von ihm) Gedachte $\leftarrow$ etwas, was (von ihm) gedacht wurde (? wird) (Passiv) - to, co pomyślat (Aktiv).

Eine Abart der oben besprochenen Konstruktion ist der Nominalisierungsrahmen "wszystko, co", der regelmäßig dann gebraucht wird, wenn die Substantivierung des Ausgangstextes mit dem Summativartikel ${ }^{8}$ alles versehen ist: alles Frühere $(\mathrm{B}, 184)$ - wszystko, co było dawniej $(\mathrm{S}, 138)$, mit allem Menschlichen (B, 246) - we wszystkim, co ludzkie (S, 183).

Eine Variante ist auch der Rahmen "coś, co", der eine Äußerung mit unbestimmter Einzelreferenz einleitet. In vielen Fällen lässt sich hier eine Konkurrenz zu den Konstruktionen vom Typ coś dobrego feststellen. Diese Konstruktion kommt dann im ZT zur Anwendung, wenn im AT der Substantivierung das Indefinitpronomen etwas vorangeht, vgl. etwas Christusmäßiges (B, 102) - coś, co upodabnia go do Chrystusa (S, 77).

Resümierend kann festgestellt werden, dass die in diesem Punkt besprochenen im Polnischen stark verbreiteten Konstruktionen, die Semantik der deutschen Substantivierungen im Polnischen sehr treu wiedergeben lassen.

\section{2. $C O S ́$ / NIC + MITTELBAR CHARAKTERISIERENDES ATTRIBUT}

Auf diese Konstruktion entfallen 5,4\% aller untersuchten Übersetzungslösungen. Dieser Typus umfasst Konstruktionen, die aus dem Pronomen coś (etwas) bzw. nic (nichts) und einem mittelbar charakterisierenden Attribut gebaut sind, z.B. coś dobrego, nic nowego. Im Polnischen sind derartige Konstruktionen stark verbreitet, vgl.:

${ }^{8}$ Der Terminus nach Weinrich (1993). 
(15) Umiejętność odwoływania się do tej wyobraźni wydaje się zatem czymś decydującym dla dalszych losów teologii. (W, 8$)$

Diese Konstruktion entspricht genau der Bedeutung der deutschen Adjektivsubstantivierungen, die durch das Indefinitpronomen etwas oder nichts eingeleitet werden (etwas Überraschendes, nichts Übles). Sie besteht aus dem Indefinitpronomen coś (oder seiner Verneinung nic) und einem Adjektivwort im Genetiv: coś dobrego, coś zjedzonego, nic ważnego. Diese Konstruktion kann auch dann eingesetzt werden, wenn die AS-Einheit eine indefinite Deskription ist (ohne Artikel bzw. mit einem unbestimmten Artikel):

(16) (...) im Zustande der Unfähigkeit, ein Besseres zu tun (...). (DF, $600)$,

(...) $\mathrm{w}$ stanie niemożności stworzenia czegoś lepszego (...). (DF, 413),

In seltenen Fällen kann das mittelbar charakterisierende Attribut eine gerundiale Form sein, vgl.:

(17) (...) denn er vermutete Essbares. $(P, 11)$

(...) spodziewal się bowiem czegoś do jedzenia. $(P, 10)$.

Wegen offensichtlicher formaler Parallelismen zwischen Deutsch und Polnisch wird diese übersetzerische Lösung quasi automatisch eingesetzt, wenn die AT-Einheit mit dem Indefinitpronomen etwas/nichts eingeleitet ist.

\section{ADJEKTIVSUBSTANTIVIERUNG}

In diesem Fall wurde eine Adjektivsubstantivierung des AT mit einer Adjektivsubstantivierung im ZT substituiert, d.h., die Wortbildungsstruktur der AS wurde in der ZS beibehalten. Diese Lösung kam in 6,6\% der Fälle zur Anwendung. Unter den in den Übersetzungen gebrauchten Substantivierungen kamen $u$. a. folgende vor: ukazane, niewymowne, nieuchwytne, najlepsze, nowe, dobre, złe, niepojmowalne. Der verhältnismäßig geringe Anteil an Substantivierungen in den untersuchten Texten ist dadurch zu erklären, dass die Substantivierung als Wortbildungsmuster im Polnischen bei der Bildung abstrakter Begriffsbezeichnungen viel seltener als im Deutschen gebraucht wird (vgl. Pkt. 2). Abweichendes lässt sich allerdings im Sprachduktus der Philosophie feststellen, was zum Teil auf das Calque-Verfahren zurückgeführt werden kann. Viele hierzu gehörende Substantivierungen können als philosophische Termini betrachtet werden. Die meisten der in den untersuchten Übersetzungen festgestellten Substantivierungen können dem philosophisch-teologischen Grenzgebiet zugeordnet werden (Boskie, ludzkie, niepojęte). Es gibt nur ganz wenige neutrale Substantivierungen mit 
abstrakter Bedeutung im Polnischen, die lexikałisiert sind: nowe, stare, złe (złe go opętato, złe nie śpi, poczytywać coś komuś za złe etc.), dobre (skłaniać się ku dobremu, doznawać dużo dobrego, prowadzić do dobrego). Teilweise sind sie dann ein fester Bestandteil von Phraseologismen: idzie nowe, podróż w nieznane, czarno na biatym, lepsze wrogiem dobrego.

Bei der Wahl dieser Übersetzungsmöglichkeit ist darauf zu achten, dass es nicht zur formalen Entlehnung kommt.

\section{READJEKTIVIERUNG}

Eine weitere gewählte Übersetzungsmöglichkeit ist die Überführung des Substantivs der AS ins Adjektiv in der ZS. Diese Art der Transposition kam relativ selten vor, denn nur in $2 \%$ aller Fälle. Was die Frequenz anbelangt, ist demnach der Typ als marginal anzusehen. Gleichwohl ist diese Lösung von Bedeutung, da sie in bestimmten Konstruktionen bevorzugt eingesetzt wird. Ziemlich regelmäßig kommt die Readjektivierung in Sätzen mit einem Kopulaverb und Prädikativ vor, wo das substantivierte Adjektivwort im Deutschen die Rolle des Trajektors, der Vordergrundfigur, spielt:

(18) Denn das Augenfällige an ihm ist nicht seine charismatische Begabung, Dinge zu sehen, die man ihm verschweigt, und andere im voraus zu wissen (...). (B, 245),

Najważniejsze u niego były nie charyzmatyczne zdolności dostrzegania spraw przed nim zatajonych lub przewidywania innych (...). $(\mathrm{S}, 183)$

Um denselben Effekt im Polnischen zu erreichen, readjektiviert der Übersetzer die Substantivierung und thematisiert dann das Adjektiv in Prädikativfunktion. So kommt es zur Umkehrung der für das Polnische typischen (unmarkierten) Thema-Rhema-Gliederung. Auf das im Thema gesetzte Adjektiv fällt der Hauptakzent, die Aufmerksamkeit des Konzeptualisierenden wird auf dieses Element fokussiert. Ähnliches passiert in folgenden Satzanfängen: Dziwne było natomiast, że... / Najwłaściwsze to milczeć. Das Adjektiv kann auch im Instrumental stehen. Der Instrumental ist beim Prädikativ im Polnischen relativ selten, Klemensiewicz (1963:35) bemerkt, dass er nur bei einigen Schriftstellern und unter speziellen stilistischen Bedingungen vorkomme. Eine der Funktionen des Instrumentals ist m.E. die Hervorhebung:

(19) Vielmehr war es das Merkwürdige und tief Bezeichnende, daß (...). (DF, 143),

Dziwnym natomiast i wielce charakterystycznym był fakt, iż (...). (DF, 101) 
Die Substantivierung kann auch in der Funktion eines Landmarks gebraucht werden, was sich automatisch in der Position des Adjektivs im Polnischen widerspiegelt:

(20) Sollte nicht auch meine eigene Beichte, (...), etwas ebenso Wesentliches werden (...). (B, 194),

Czy spowiedź, (...), nie moglaby także stać się równie decydującą (...). (S, 146)

Die Readjektivierung kann zu Änderungen im kreierten Textbild führen. Auch im nächsten Beispiel dient die Substantivierung dem Effekt des InVordergrund-Stellens, sie ist TR der Phrase, während Gnade als Attribut die Funktion des LM erfüllt. Es wäre an der Stelle auch eine konventionelle Lösung möglich (um von der unverhältnismäßigen Gnade getroffen zu werden), der Konzeptualisierende entscheidet sich aber für eine weniger konventionelle Konstruktion, um eben dieses Unverhältnismäßige hervorzuheben. Dies geht in der Übersetzung verloren:

(21) Und eben das war der rechte Auftakt, um vom Unverhältnismäßigen der Gnade getroffen zu werden. (B, 205),

I to właśnie jest stosowny moment doświadczenia przeobfitej laski. $(\mathrm{S}, 154)$

Beim Einsetzen dieses Übersetzungstyps ist folglich zu beachten, dass die Readjektivierung zu semantischen Änderungen im Textbild führen kann, vor allem zu seiner Konventionalisierung.

\section{ADVERBIALISIERUNG}

Die Transposition einer Substantivierung zum Adverb kommt selten vor (1,5\% der Fälle) und ist meistens Folge der paraphrastischen Übersetzung des ganzen Satzes. Die Adverbialisierung kann notwendig werden, wenn im ZT ein synonymes Verb eingesetzt wird, das als obligatorische Ergänzung ein Adverb hat, wie im folgenden Beispiel:

(22) Das ist das Gewöhnliche. (B, 115)

Tak bywa najczęściej. $(S, 87)$

\section{TRANSPOSITION ZUM VERB}

Die Transposition der Substantivierung zum Verb wird von der Konzentration begleitet. In Folge der Konzentration wird das Verb, in der Regel das Kopulaverb sein, reduziert. Dieser Typ kommt vorwiegend bei Substan- 
tivierungen vor, derer Basis ein deverbales Adjektivwort ist. Die Substantivierung wird auf das Basisverb zurückgeführt, das im ZT die Substantivierung und das Kopulaverb ersetzt:

(23) Und das weitere Aufregende ist (...). (B, 150)

Irytuje również to (...). (S, 113)

(24) Überall ist die Demut das Entscheidende (...). (B, 174)

(...) wszędzie decyduje pokora (...). (S, 131)

Dieser Typ ist als marginal zu werten. Es kommt fast ausschließlich bei den Substantivierungen in Kopulasätzen vor. Im untersuchten Material machte er einen Prozent aller Entscheidungen aus. Sein Einsatz führt darüber hinaus zu Änderungen im Textbild, die Figur wird reduziert, während die Relation stärker betont wird.

\section{ANDERE MÖGLICHKEITEN}

Vereinzelt wurden auch andere Äquivalente eingesetzt, die jedoch als unwesentlich zu betrachten sind. In der Übersetzung kann beispielsweise eine Substantivierung pronominalisiert oder mit einem Satz "umschrieben" werden, des Weiteren sind morphologische Relativierung oder Etikett und syntaktische Relativierung zu nennen. Pronominalisierung wird dann eingesetzt, wenn die Substantivierung im Text wiederholt wird, oder redundant ist, weil sie das Vorhergehende noch einmal zusammenfasst, wie im folgenden Satz:

(25) Schreibt man aber alles auf und liest man einfach das Geschriebene herunter, so ist das nicht mehr ein spontanes Bekenntnis (...). (B, 121), Ieśli się jednak napisze wszvstko i potem to zwyczajnie odczyta, spowiedź utraci swój spontaniczny charakter (...). (S, 92)

Die Umwandlung in einen Satz geht i.d.R. mit Amplifikation einher:

(26) (...) wählt er die Sünde als das zu Zeigende (B, 20)

Jego wybór padl na grzech. Pragnie go ukazać człowiekowi. (S, 17)

\section{SCHLUSSBEMERKUNGEN}

Zusammenfassend lässt sich feststellen, dass die fünf wichtigsten Äquivalenztypen für die substantivierten adadjektivischen Abstrakta im Polnischen die folgenden sind: (1) Ableitungen (36,7\%) - nowość, (2) Adjektiv und Etikett $(23,9 \%)$ - rzeczy nowe, (3) Indefinitpronomen coś/nic + mittelbar charakterisierendes Attribut (12,2\%) - coś nowego, (4) Nominalisierungsrahmen 
to, co... + Adjektiv (10,8\%) - to, co nowe, (5) Substantivierung $(6,6 \%)-$ nowe. Diese Zahlen illustriert das Schaubild:

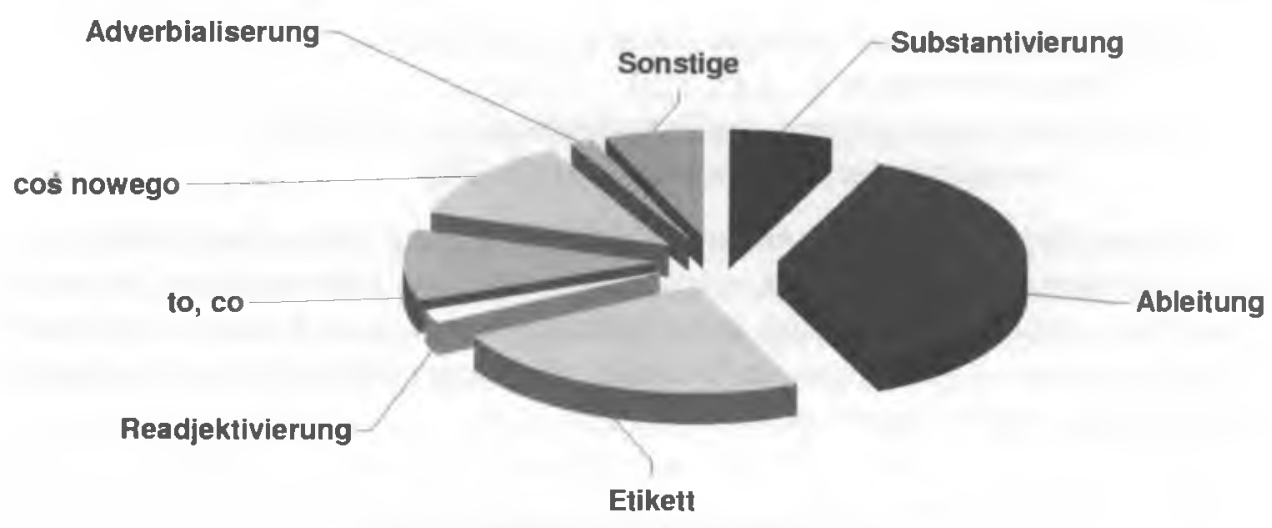

Addiert man diese Prozentzahlen, so kommt man auf folgendes Ergebnis: nur etwa bei einem Fünftel der Lösungen kann von einer relativen Symmetrie gesprochen werden. In $80 \%$ der Fälle ist eine Asymmetrie festgestellt worden, was bedeutet, dass dieser Substantivierungstyp eine potentielle Quelle der Übersetzungsschwierigkeiten sein kann. Diese Annahme bestätigt auch die didaktische Praxis, in der Studenten bei den Übersetzungen häufig über die deadjektivischen substantivierten Abstrakta stolpern und an ihnen scheitern.

\section{BIBLIOGRAPHIE}

Bonhoeffer, D., 1983. Nachfolge. München: Chr. Kaiser Verlag.

Bonhoeffer, D., 1997. Nasladowanie. Übers. v. J. Kubaszczyk. Poznan: W drodze.

Grabowski, M., 1995. Na obrzezach sacrum. Poznań: W drodze.

Hildebrand, von D., 1969. Sittliche Grundhaltungen. Regensburg: Verlag Josef Habbel.

Hildebrand, von D., 1982. Fundamentalne postawy moralne. In: Wobec wartosici. Poznan: W drodze.

Mann, T., 1991. Doktor Faustus. Das Leben des deutschen Tonsetzers Adrian Leverkühn erzählt von einem Freunde. Fischer Taschenbuchverlag.

Mann, T., 1985. Doktor Faustus. Zywot niemieckiego kompozytora Adriana Leverkühna, opowiedziany przez jego przyjaciela. Übers. v. Maria Kurecka und Witold Wirpsza. Warszawa: Czytelnik.

Speyr, von A., 1960. Die Beichte. Einsiedeln.

Speyr, von A., 1993. Spowiedż. Übers. v. Wiesław Szymona OP. Poznañ: W drodze.

Süskind, P., 1985. Das Parfum. Die Geschichte eines Mörders. Zürich.

Süskind, P., 1996. Pachnidło. Historia pewnego mordercy. Übers. v. Małgorzata Eukasiewicz. Warszawa.

Węcławski, T., 1995. W teologii chodzi o Ciebie. Kraków: Znak. 


\section{SEKUNDÄRLITERATUR}

Grzegorczykowa, R., 1979. Zarys stowotwórstwa polskiego. Warszawa: PWN.

Jadacka, H., 2001. System słowotwórczy polszczyzny. Warszawa: Wydawnictwo Naukowe PWN. Jodlowski, S., 1964. Substantywizacja przymiotnikow w języku polskim. Wrocław, Warszawa, Kraków: ZNiO.

Klemensiewicz, Z., 1963. Zarys składni polskiej. Warszawa: PWN.

Kubaszczyk, J.: Substantywizacje $w$ przekładzie. Niemiecko-polskie studium kontrastywne. Poznań 2003 (unveröffentlichte Dissertation).

Kubaszczyk, J., 2004. Übersetzung substantivierter Adjektive als Sachnamen ins Polnische mit Hilfe eines Etiketts, Convivium, S. 245-262.

Langacker, Ronald W., 1991. Concept, Image, and Symbol. The Cognitive Basis of Grammar. Berlin/New York.

Malblanc, A., 1961/1968. Stylistique comparée du français et de l'allemand. Paris.

Taylor, J.R., 2002. Cognitive Grammar. Oxford: University Press.

Vinay, J.-P. /Darbelnet, J., 1958/60. Stylistique comparée du français et de l'anglais. Paris.

Weinrich, H., 1993. Textgrammatik der deutschen Sprache. Mannheim: Dudenverlag.

SIGEL

B = SPEYR, VON A.: Die Beichte.

$S=$ SPEYR, VON A.: Spowiedź.

DF = MANN, T.: Doktor Faustus (dt. und poln.).

$P=$ SÜSKIND, P.: Das Parfum / Pachnidlo.

$\mathrm{W}=$ WĘCŁAWSKI, T., 1995. W teologii chodzi o Ciebie. 